\title{
$\mathbf{A}_{a} \mathbf{D}_{d}$ 型氢键流体的统计理论( II)：氢键 网络的统计特征 ${ }^{*}$
}

\author{
王海军 ${ }^{* *}$
}

(1) 河北大学化学与环境科学学院, 保定 071002; (2) 中国科学院国际材料物理中心, 沈阳 110016)

\begin{abstract}
摘要 基于 $\mathrm{A}_{a} \mathrm{D}_{d}$ 型氢键体系中氢键簇合物数量分布函数的不变特征, 给出临界点后溶胶相和 凝胶相的平衡自由能的解析形式. 进而利用分布函数的渐近形式研究了凝胶自由能和凝胶相中 的氢键数在临界点附近的标度行为, 给出二者所满足的标度律. 进一步分析凝胶网络结构中的分 子间氢键和分子内氢键的相关特征, 给出体系分子内氢键的数量与体系氢键度之间的定量关系.
\end{abstract}

\section{关键词氢键网络凝胶相 标度律}

氢键网络是指组成体系的结构单元之间由氢键 联结而成的一种特殊结构 ${ }^{[1]}$, 其广泛存在于自然界中, 冰就是一种常见的氢键网络. 一些特殊的氢键网络 对诸如蛋白质和核酸等很多物质的结构和功能有着 重要影响, 并在生命科学中起着关键作用. 例如, 打 开DNA中的氢键网络使其解旋, 然后以两条单链为 模板分别进行复制, 可重新组成两个与原来DNA结 构完全相同的氢键网络, 生命的遗传信息籍此得以 传递. 单个氢键的寿命十分短促, 而氢键网络中大量 分子内氢键却可以使得氢键网络的寿命显著增加, 以维持其相应的稳定状态. 在此意义上讲, 氢键网络 的存在是分子内氢键之间的一种集体合作现象.

迄今, 实验方面对于氢键网络的研究多集中于
应用核磁共振、红外和拉曼光谱、X-光和中子衍射等 实验手段进行. 理论研究方面, 常用的方法包括分子

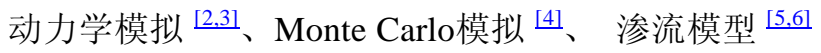
和可逆凝胶化理论等 ${ }^{[7 ~ 10]}$, 这些方法对于相关体系的 网络结构都有不同程度的研究. 有关氢键网络应用 方面的工作涉及到多个领域，尤其是利用氢键所进 行的超分子组装 ${ }^{[11,12]}$ 和氢键致液晶 ${ }^{[13]}$ 等方面的工作 富有特色, 已成为近年来物理、化学、材料科学和工 程等领域的研究热点. 其中基于氢键组装成的各类 膜材料正是利用了分子内氢键的合作性. 因此研究 氢键网络的结构特征有助于深入了解氢键的特殊功 能和作用.

对于氢键液体或溶液而言, 当溶胶-凝胶相变发 
生后, 凝胶相中开始出现分子内氢键, 形成氢键网络. 本文基于前文对 $\mathrm{A}_{a} \mathrm{D}_{d}$ 型氢键体系的探讨 ${ }^{[14]}$, 集中对 凝胶相的统计特征进行讨论. 主要研究了临界点后 溶胶相和凝胶相的平衡自由能问题以及凝胶自由能 的标度行为. 进一步就凝胶网络中的分子内氢键数 和分子间氢键数进行分析, 给出它们随体系氢键度 的变化情况. 在整个分析过程中, 我们认为溶胶相中 的分子内氢键数很少, 并因此忽略溶胶相中的分子 内氢键. 一般情况下，研究与各种不同尺寸簇合物有 关的平均物理量的标度行为需要考察数量分布函数 在临界点附近的渐近行为 [15], 为此文中首先讨论数 量分布函数的渐近表达式, 然后指出凝胶自由能和 凝胶中的氢键数在临界点附近具有同样的标度行为, 并给出相应的标度律.

\section{1 氢键簇合物的数量分布函数的渐近形式}

考虑溶胶相中氢键簇合物以类树方式联结而成, 并忽略氢键簇中的分子内氢键, 由此可得氢键簇合

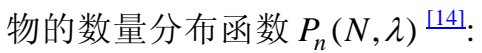

$$
P_{n}(N, \lambda)=\frac{g_{n}}{n !}\left(P_{1}\right)^{n}\left[\frac{\lambda}{(a N-\lambda)(d N-\lambda)}\right]^{n-1},
$$

式中 $\lambda$ 是此时体系中的平衡氢键数, $g_{n}$ 是一个组合 因子，描述了由 $n$ 个结构单元所形成的氢键簇合物的 各种可能构型. $P_{1}=N\left(1-\frac{\lambda}{a N}\right)^{a}\left(1-\frac{\lambda}{d N}\right)^{d}$, 是平衡 时体系中的单体 (未参与形成氢键的分子)数. 通过分 布函数可定义临界点前体系的 $k$ 次数量矩为

$$
M_{k}=\sum_{n} n^{k} P_{n}(N, \lambda), \quad(k=0,1,2,3 \ldots),
$$

其中零次矩的物理意义是给出了体系中的分子数, 一次矩所描述的是体系总的结构单元数, 即 $M_{0}=(N-\lambda)$ 和 $M_{1}=N$. 进一步由 $k$ 次矩的定义出 发, 可得 $(k+1)$ 次矩和 $k$ 次矩之间满足如下递推关系:

$$
M_{k+1}=\frac{1}{D}\left[\left(a d N^{2}-\lambda^{2}\right)+\lambda(a N-\lambda)(d N-\lambda) \frac{\partial}{\partial \lambda}\right] M_{k},
$$

其中因子 $D$ 的形式为

$$
D=a d(N-\lambda)^{2}-(a-1)(d-1) \lambda^{2} .
$$

容易证明零次矩和一次矩满足此递推关系. 进 而以一次矩为起始循环点, 可以计算体系的高次矩. 其中二次矩为 $M_{2}=\frac{1}{D}\left(a d N^{2}-\lambda^{2}\right) N$, 由此可以看出 当 $D=0$ 时, 体系的二次矩发散, 相应的二次矩以上 的高次矩的发散行为表现为 $M_{k} \propto D^{2 k-3}$. 因此条件 $D=0$ 意味着体系溶胶-凝胶几何相变的开始, 与此 相应的临界氢键数 $\lambda_{\mathrm{c}}$ 满足方程

$$
\frac{\lambda_{\mathrm{c}}}{N}=\frac{\sqrt{a d}}{\sqrt{a d}+\sqrt{(a-1)(d-1)}} .
$$

一旦溶胶-凝胶相变发生 $\left(\lambda \geqslant \lambda_{\mathrm{c}}\right)$, 凝胶相出现, 使得描述体系性质的一些平均物理量也发生相应变 化. 因为在临界点前体系的一些平均物理量都是相 对于有限大小的氢键簇合物的平均, 所以凝胶点后 应考虑这些物理量相对于溶胶相的平均. 进而通过 研究临界点前后这些物理量的关联, 可以更好地描 述几何相变的本质. 按照李泽生等的建议 ${ }^{[16 ~ 18]}$, 利 用平均物理量的不变特征可以大大简化临界点后体 系性质的研究. 为此, 当 $\lambda>\lambda_{\mathrm{c}}$ 时, 需要引入溶胶变 量. 为方便起见, 现以 $N^{\prime}$ 和 $\lambda^{\prime}$ 分别表示临界点后溶 胶相中的结构单元数和氢键数. 进一步根据数量分 布函数的不变性 ${ }^{[14]}$, 可以导出平均物理量的不变特 征 ${ }^{[16 ~ 18]}$. 平均物理量的不变特征指出, 对一个仅与 指标 $n$ 有关、而与 $\lambda$ 无关的物理量 $H_{n}$ 而言，在临界 点前, 若其平均值表示为 $H(N, \lambda)=\sum_{n} H_{n} P_{n}(N, \lambda)$, 则在临界点后仅需将临界点前该平均物理量表示式 中的体系变量代换为临界点后相应的溶胶变量, 即 可得到其在临界点后的表示式，而函数形式保持不 变. 作为此理论的直接应用, 可得体系在临界点后的 零次矩 $M_{0}^{\prime}$, 一次矩 $M_{1}^{\prime}$ 和二次矩 $M_{2}^{\prime}$ 以及相应的递推 方程分别为

$$
\begin{aligned}
& M_{0}^{\prime}=N^{\prime}-\lambda^{\prime}, M_{1}^{\prime}=N^{\prime}, M_{2}^{\prime}=\frac{N^{\prime}}{D^{\prime}}\left(a d N^{\prime 2}-\lambda^{\prime 2}\right), \\
& M_{k+1}^{\prime}= \\
& \frac{1}{D^{\prime}}\left[\left(a d N^{\prime 2}-\lambda^{\prime 2}\right)+\lambda^{\prime}\left(a N^{\prime}-\lambda^{\prime}\right)\left(d N^{\prime}-\lambda^{\prime}\right) \frac{\partial}{\partial \lambda^{\prime}}\right] M_{k}^{\prime},
\end{aligned}
$$

其中因子 $D^{\prime}$ 的形式为 


$$
D^{\prime}=\operatorname{ad}\left(N^{\prime}-\lambda^{\prime}\right)^{2}-(a-1)(d-1) \lambda^{\prime 2} .
$$

在临界点附近, 因子 $D$ 和 $D^{\prime}$ 满足条件 $D \rightarrow 0$ 和 $D^{\prime} \rightarrow 0$. 而在临界点后, 体系和溶胶中的氢键数分 别满足条件 $\lambda \geqslant \lambda_{\mathrm{c}}$ 和 $\lambda^{\prime} \leqslant \lambda_{\mathrm{c}}$. 当体系处于临界点附 近时，体系的平均物理量应以相应的渐近形式表达. 具体到临界点前的 $k$ 次矩 $M_{k}$ 和临界点后的 $k$ 次矩 $M_{k}^{\prime}$ 而言, 在临界点附近, 二者可统一用渐近的 $k$ 次 矩 $\tilde{M}_{k}$ 来表示. 当 $k \geqslant 2$ 且 $D \rightarrow 0, D^{\prime} \rightarrow 0$ 时, 渐近的 $k$ 次矩可表示为 $\tilde{M}_{k}=\frac{I_{k}}{|D|^{2 k-3}}$. 进而利用上面的递推 公式(2), 可以发现物理量 $I_{k+1}$ 和 $I_{k}$ 满足如下循环关 系:

$$
I_{k+1}=-(2 k-3) \lambda_{\mathrm{c}}\left(a N-\lambda_{\mathrm{c}}\right)\left(d N-\lambda_{\mathrm{c}}\right)\left(\frac{\mathrm{d} D}{\mathrm{~d} \lambda}\right)_{D=0} I_{k},
$$

其中 $I_{2}=N\left(a d N^{2}-\lambda_{\mathrm{c}}^{2}\right)$. 进而以 $I_{2}$ 为起始点, 可以获 得高次矩在临界点附近的渐近形式. 相应地, 在临界 点附近, 数量矩定义中的数量分布函数也须用渐近 分布 $\tilde{P}_{n}$ 来代替. 根据数量矩的定义并将求和化为积 分形式, 可得

$$
\begin{gathered}
\tilde{M}_{k}=\frac{I_{k}}{|D|^{2 k-3}}=\int n^{k} \tilde{P}_{n} \mathrm{~d} n, \\
\tilde{M}_{k+1}=\frac{I_{k+1}}{|D|^{2 k-1}}=\int n^{k+1} \tilde{P}_{n} \mathrm{~d} n,(k \geqslant 2,|D| \rightarrow 0) .
\end{gathered}
$$

由此方程开始, 利用唐敖庆和李泽生等人报道的方 法 ${ }^{[15,19]}$, 考虑二次以上的数量矩, 可以得到体系的渐 近分布函数为

$$
\tilde{P}_{n}=\mathrm{BNn}^{-\tau} \exp \left(-\frac{n}{n_{\mathrm{c}}}\right),
$$

其中 $\mathrm{B}$ 是一个常数, $\tau=\frac{5}{2}$, 是氢键簇合物的临界指 数. 而其中的 $n_{\mathrm{c}}$ 是体系的临界关联尺度, 具体形式为

$$
n_{\mathrm{c}}=\frac{\left(a N-\lambda_{\mathrm{c}}\right)\left(d N-\lambda_{\mathrm{c}}\right)}{\operatorname{adN}\left(N-\lambda_{\mathrm{c}}\right)} \xi^{-\frac{1}{\sigma}},
$$

此式中的 $\xi=\frac{\left|\lambda-\lambda_{\mathrm{c}}\right|}{\lambda_{\mathrm{c}}}$, 表示体系与临界点接近的程度. $\sigma=\frac{1}{2}$, 是关联长度的临界指数. 当 $\xi \rightarrow 0^{+}$时 $\left(0^{+}\right.$是 无限小的正实数), $n_{\mathrm{c}} \rightarrow \infty$, 从而表明当溶胶-凝胶相 变发生时, 体系的关联长度显著增加.

从方程(10)中所给出的数量分布函数的渐近表 达式中可知函数 $\exp \left(-\frac{n}{n_{\mathrm{c}}}\right)$ 实际上起到了截断函数的 作用. 需要指出的是, 在临界点附近, 由此渐近分布 所导出的二次矩和高次矩的值与通过标准定义所计 算的值完全一致. 而当以其计算体系的一次矩时, 计 算结果需要经过校正才可与一次矩的物理意义对应 起来 ${ }^{[15]}$.

\section{2 凝胶相的自由能及其标度律}

前文 ${ }^{[14]}$ 已给出体系在压强为 $P$ 、体积为 $V$ 、温 度为 $T$ 时因氢键形成所致的平衡自由能 $F_{\mathrm{eq}}(N, \lambda)$ 为

$$
F_{\text {eq }}(N, \lambda)=k_{\mathrm{B}} T\left[\lambda+N \ln \left[\left(1-\frac{\lambda}{a N}\right)^{a}\left(1-\frac{\lambda}{d N}\right)^{d}\right]\right],
$$

其中 $k_{\mathrm{B}}$ 是 Boltzmann 常数. 上式表明与平衡自由能直 接相关的物理量是平衡氢键数 $\lambda$, 它满足方程 $\frac{\lambda}{(a N-\lambda)(d N-\lambda)}=\frac{v_{\mathrm{hb}}}{V} \exp \left(-\frac{\varepsilon}{k_{\mathrm{B}} T}\right)$, 此式中的 $v_{\mathrm{hb}}$ 表 示成键体积, $\varepsilon$ 表示氢键的成键自由能. 方程(12)给 出了体系因氢键所致的自由能的变化, 在其他条件 确定时仅与平衡的氢键数 $\lambda$ 有关. 从前文导出的该 物理量的过程可知方程(12)在溶胶-凝胶相变临界点 前后都适用.

在临界点前, 体系只有溶胶相, 所以当 $\lambda<\lambda_{\mathrm{c}}$ 时, 方程(12)中的平衡自由能即为溶胶相的平衡自由能. 直接应用平均物理量的不变特征, 可得到临界点后 溶胶相的平衡自由能 $F_{\mathrm{eq}}^{\mathrm{sol}}\left(N^{\prime}, \lambda^{\prime}\right)$ 为

$$
\begin{gathered}
F_{\mathrm{eq}}^{\mathrm{sol}}\left(N^{\prime}, \lambda^{\prime}\right)=k_{\mathrm{B}} T\left[\lambda^{\prime}+N^{\prime} \ln \left[\left(1-\frac{\lambda^{\prime}}{a N^{\prime}}\right)^{a}\left(1-\frac{\lambda^{\prime}}{d N^{\prime}}\right)^{d}\right]\right], \\
\left(\lambda \geqslant \lambda_{\mathrm{c}}\right) .
\end{gathered}
$$

体系在临界点后只有溶胶相和凝胶相, 相应的 总自由能是此两相自由能之和, 因此凝胶自由能满 
足方程 $F_{\mathrm{eq}}^{\mathrm{gel}}=F_{\mathrm{eq}}(N, \lambda)-F_{\mathrm{eq}}^{\mathrm{sol}}\left(N^{\prime}, \lambda^{\prime}\right)$, 计算可得

$$
\begin{aligned}
F_{\mathrm{eq}}^{\mathrm{sol}}\left(N^{\prime}, \lambda^{\prime}\right)=k_{\mathrm{B}} T & {\left[\left(\lambda-\lambda^{\prime}\right)+N \ln \frac{P_{1}}{N}-N^{\prime} \ln \frac{P_{1}}{N^{\prime}}\right], } \\
\left(\lambda \geqslant \lambda_{\mathrm{c}}\right), &
\end{aligned}
$$

其中 $P_{1}\left(N^{\prime}, \lambda^{\prime}\right)=N^{\prime}\left[\left(1-\frac{\lambda^{\prime}}{a N^{\prime}}\right)^{a}\left(1-\frac{\lambda^{\prime}}{d N^{\prime}}\right)^{d}\right]$, 是临界 点后体系中的单体数, 亦即未参与形成氢键的自由 分子数, 且 $P_{1}\left(N^{\prime}, \lambda^{\prime}\right)=P_{1}(N, \lambda)$.

根据方程(13)和(14)可知, 在临界点以前, 因为 $N=N^{\prime}, \lambda=\lambda^{\prime}$, 所以体系中凝胶和溶胶部分自由能 在临界点前分别为

$$
F_{\text {eq }}^{\text {gel }}=0, F_{\text {eq }}^{\text {sol }}=k_{\mathrm{B}} T\left(\lambda+N \ln \frac{P_{1}}{N}\right),\left(\lambda \leqslant \lambda_{\mathrm{c}}\right) .
$$

注意到临界点前体系没有凝胶相, 凝胶自由能自然 为零, 所以体系和溶胶的氢键自由能必然相等, 这与 实际的物理情况相符.

在临界点后体系满足 $\lambda>\lambda_{\mathrm{c}}$ 和 $\lambda^{\prime}<\lambda_{\mathrm{c}}$ 两个条件, 此时 $\xi=\frac{\lambda-\lambda_{\mathrm{c}}}{\lambda_{\mathrm{c}}}=\frac{\lambda_{\mathrm{c}}-\lambda^{\prime}}{\lambda_{\mathrm{c}}}$. 在体系处于临界点附近时, 意味着 $\xi$ 是一个正的无限小量, 即 $\xi \rightarrow 0^{+}$. 由方程 (14)可知, 在临界点后, 凝胶自由能不仅与凝胶中的 氢键键数有关, 也与单体数有关. 为了探讨临界点附 近的凝胶自由能的相关特征，须对其中各项进行相 应分析. 按照假设, 在临界点前, 体系中的氢键都是 分子间氢键，亦即溶胶相中的氢键簇具有类树状结 构, 而凝胶相的出现则表示体系中最大的氢键簇合 物中开始出现分子内氢键, 即环键. 当 $\xi \rightarrow 0^{+}$时, 此 时体系中的环键数目依然很少, 加之凝胶分子可被 视作体系中最大的单个分子簇, 因此当条件 $\xi \rightarrow 0^{+}$ 满足时, 凝胶中的氢键数 $\left(\lambda-\lambda^{\prime}\right)$ 趋近于 $\left(N-N^{\prime}\right)$. 为 了更好地描述临界点后的溶胶相和凝胶相的相关特 征, 须引入溶胶分数 $S$ 和凝胶分数 $G$ 的概念, 它们满 足方程 $S+G=1$, 其中 $S=\frac{N^{\prime}}{N}$. 由此可知凝胶自由能 中的项 $\left(N \ln \frac{P_{1}}{N}-N^{\prime} \ln \frac{P_{1}}{N^{\prime}}\right)$ 可以重新表示为
$\left(N-N^{\prime}\right) \ln \frac{P_{1}}{N}+N^{\prime} \ln S$. 由于 $\xi \rightarrow 0^{+}$时, $S \rightarrow 1$, 所以 凝胶自由能在临界点附近有如下渐近形式:

$$
F_{\mathrm{eq}}^{\mathrm{gel}} \rightarrow N k_{\mathrm{B}} T G\left[1+\ln \left(\frac{P_{1}}{N}\right)\right], \quad\left(\xi \rightarrow 0^{+}\right) .
$$

此式表明, 凝胶中的氢键数和凝胶自由能在临界点 附近的渐近行为相似. 同时, 因为在临界点处体系中 的单体数有限, 所以 $\ln \left(\frac{P_{1}}{N}\right)$ 有限. 因此凝胶自由能 和凝胶中的氢键数的标度行为与凝胶分数 $G$ 的标度 行为一致.

在临界点附近，溶胶分数 $S$ 用渐近分布可表示 为

$$
S=\mathrm{B} \int_{1}^{\infty} n^{1-\tau} \exp \left(-\frac{n}{n_{\mathrm{c}}}\right) \mathrm{d} n \cong \mathrm{B} \int_{1}^{n_{\mathrm{c}}} n^{1-\tau} \mathrm{d} n \cong 1-\mathrm{B}^{\prime} \xi^{\frac{\tau-2}{\sigma}},
$$

上式中 $B^{\prime}$ 是一个常数. 在计算过程中已经利用了截 断函数的作用. 考虑到临界点前溶胶分数恒为 1 , 因 此最终的积分结果已经经过一个常数因子的校正, 以使结果符合物理要求。上式表明在临界点附近, $G \sim \xi^{\frac{\tau-2}{\sigma}}$, 亦即, $F_{\mathrm{eq}}^{\mathrm{gel}} \sim \xi^{\frac{\tau-2}{\sigma}}$. 另外, 在临界点附近 将溶胶分数展开, 同时忽略高阶小量, 可得 $G \sim \xi^{\beta}$, 其中 $\beta$ 为凝胶分数在临界点附近的临界指数, 且 $\beta=1$. 由此可知 $F_{\mathrm{eq}}^{\mathrm{gel}} \sim \xi^{\beta}$, 综合这些结果, 可以发 现凝胶自由能和凝胶中的氢键数在临界点附近所满 足的标度律为

$$
\sigma \beta=\tau-2 .
$$

上式表明凝胶自由能和凝胶分数的标度律形式相同. 事实上，尽管在平均场理论的框架下所给出的临界 指数并不十分准确, 而标度律的正确性已为大量计 算机模拟的结果所证实.

\section{3 氢键凝胶网络的统计特征}

溶胶-凝胶相变发生后，凝胶分数逐渐增加，很 多溶胶中的氢键簇合物开始与凝胶联结, 致使凝胶 中的结构单元数增加. 与溶胶相中类树状结构相比, 凝胶相中的氢键簇中开始出现分子内氢键(环键), 而 
且随着体系中氢键数的增多, 凝胶分子中的环键数 目也相应增加. 相应地, 整个体系的统计特征由于凝 胶相的出现也发生变化. 这主要源于分子内氢键数 的增加导致了氢键网络结构的形成，伴随有体系中 的弹性有效交联点和弹性有效链节开始增多以及弹 性模量增大等特征 [20]. 因此讨论凝胶网络中的环键 数和树键数有助于于深入了解氢键网络的结构和功 能.

考虑在临界点后某一时刻体系中总的氢键数为 $\lambda$, 而相应的溶胶相和凝胶相中氢键数分别为 $\lambda_{\mathrm{sol}}$ 和 $\lambda_{\text {gel }}$, 则有

$$
\lambda=\lambda_{\text {sol }}+\lambda_{\text {gel }} .
$$

若以 $\lambda^{\text {tree }}, \lambda_{\text {sol }}^{\text {tree }}, \lambda_{\text {gel }}^{\text {tree }}$ 和 $\lambda^{\text {loop }}, \lambda_{\text {sol }}^{\text {loop }}, \lambda_{\text {gel }}^{\text {loop }}$ 分别表 示体系、溶胶和凝胶中的树键和环键数, 则可得如下 方程:

$$
\lambda^{\text {tree }}=\lambda_{\text {sol }}^{\text {tree }}+\lambda_{\text {gel }}^{\text {tree }}, \quad \lambda^{\text {loop }}=\lambda_{\text {sol }}^{\text {loop }}+\lambda_{\text {gel }}^{\text {loop }} .
$$

此外, 对于溶胶和凝胶中的氢键数, 也有如下方程成 立:

$$
\lambda_{\text {sol }}=\lambda_{\text {sol }}^{\text {tree }}+\lambda_{\text {sol }}^{\text {loop }}, \quad \lambda_{\text {gel }}=\lambda_{\text {gel }}^{\text {tree }}+\lambda_{\text {gel }}^{\text {loop }} .
$$

按照本文的假设, 溶胶中的分子内氢键数可被忽略, 即 $\lambda_{\mathrm{sol}}^{\text {loop }}=0$. 同时, 注意到凝胶中的结构单元数为 $N-N^{\prime}$, 而凝胶又可被视作单个氢键网络, 因此凝胶 中的树键树的数目为 $\lambda_{\text {gel }}^{\text {tree }}=N-N^{\prime}$, 由此可得凝胶中 (也是整个体系中) 的环键的数目近似为

$$
\lambda^{\text {loop }}=\lambda_{\text {gel }}^{\text {loop }}=\left(\lambda-\lambda^{\prime}\right)-\left(N-N^{\prime}\right) .
$$

由此可知在体系将发生相变时 $\left(\lambda \rightarrow \lambda_{\mathrm{c}}\right)$, 由于关系 式 $\lambda^{\prime} \rightarrow \lambda$ 和 $N^{\prime} \rightarrow N$ 成立, 所以此时体系中没有环键, 即 $\lambda_{\mathrm{c}}^{\text {loop }} \rightarrow 0$, 这与前面分析的结果一致.

为了进一步给出体系中的环键数随着体系总的 氢键数的变化关系, 必须讨论溶胶中氢键数和体系 氢键数的关系. 根据该体系的溶胶-凝胶分配方程 ${ }^{[20]}$, 可知体系的溶胶分数为 $S=X^{a} Y^{d}$, 其中的 $X$ 和 $Y$ 分 别是体系中任意一个质子受体和给体与溶胶相联的 概率. 根据概率分析可知它们满足方程

$$
X=1-\frac{\eta}{a}+\frac{\eta}{a} X^{a} Y^{d-1}, Y=1-\frac{\eta}{d}+\frac{\eta}{d} X^{a-1} Y^{d},
$$

其中物理量 $\eta$ 是体系的平衡氢键度, 定义为 $\eta=\frac{\lambda}{N}$. 再根据溶胶-凝胶分配理论 ${ }^{[21]}$, 可得溶胶中和体系中 的氢键数的关系为

$$
\lambda^{\prime}=\lambda X^{2 a-1} Y^{2 d-1} \text {. }
$$

综合以上结果, 可得体系中的环键数可以表示为

$$
\lambda^{\text {loop }}=\lambda\left(1-X^{2 a-1} Y^{2 d-1}\right)-N\left(1-X^{a} Y^{d}\right) .
$$

通过方程(23)可以看出, 对于少数非线性氢键体 系, 可以直接得到 $X$ 和 $Y$ 两个量与 $\lambda$ 之间的解析表 达式, 而对于更一般的体系, 只能通过相应的数值计 算来得到体系的分子内氢键数与体系氢键数的关系. 为了说明问题起见, 现以 $A_{2} D_{2}$ 体系为例来计算临界 点后体系的环键数. 该体系的临界氢键度为 $\eta_{\mathrm{c}}=\frac{2}{3}$, 且有 $X=Y$ 和 $X=1-\frac{\eta}{2}+\frac{\eta}{2} X^{3}$. 由此方程可知, 在临 界点前, $X=1$, 而在临界点后, $X=\left(\sqrt{\frac{2}{\eta}-\frac{3}{4}}-\frac{1}{2}\right)$. 因此凝胶相中的分子内氢键数与体系氢键数的关系 变成 $\lambda^{\text {loop }}=\lambda\left(1-X^{6}\right)-N\left(1-X^{4}\right)$. 若定义环键分数 为 $f_{\text {loop }}=\frac{\lambda^{\text {loop }}}{\lambda}$, 则有

$$
f_{\text {loop }}=\left(1-X^{6}\right)-\frac{1}{\eta}\left(1-X^{4}\right) .
$$

图 1 所示为 $\mathrm{A}_{2} \mathrm{D}_{2}$ 体系中的环键分数随着体系氢 键度的变化情况. 从中可以看出, 在理想情况下, 环 键分数接近它的极限值 $\frac{1}{2}$. 然而体系的氢键度实际 上受到外部温度、压力和体积等条件的限制, 不可能 达到完全成键的程度，因此相应的环键分数也受到 限制. 环键的数量对体系的弹性和黏性都有一定影 响, 因此计算环键数是深入了解网络结构相关性能 的基础. 结合本文结果与以前对凝胶相的其他统计 参数的研究 ${ }^{[20]}$, 可对氢键网络给出较为完备的统计 表征. 应该指出的是, 以上讨论都是基于对高分子固 化体系研究 ${ }^{[22]}$ 而进行的, 高分子中联结结构单元的 是具有较大键能的共价键或离子键, 而氢键的键能 则很低, 因此容易被破坏, 然而这也正是氢键在生命 


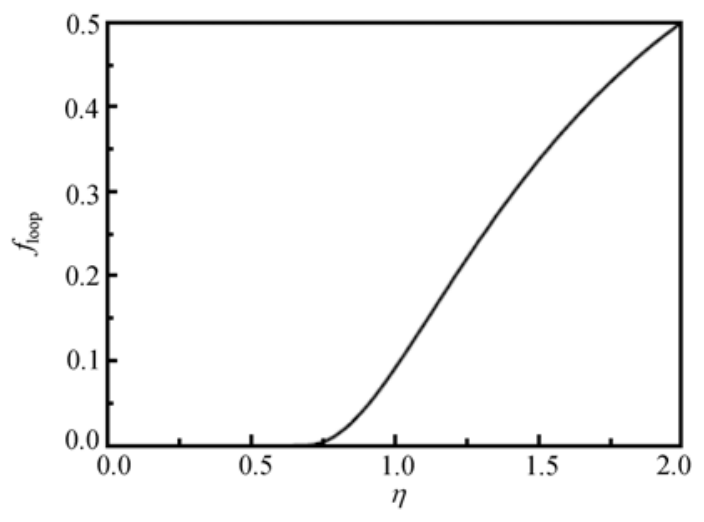

图 $1 \mathrm{~A}_{2} \mathrm{D}_{2}$ 体系中环键分数随体系氢键度的变化

科学中有重要作用的原因之一. 另外一个重要差别 是高分子凝胶网络一般呈现无序特征，而氢键网络 由于氢键的方向性则多呈现有序结构.

\section{4 结果和讨论}

以上我们从体系的平衡氢键自由能出发, 利用 数量分布函数的不变特征对临界点后溶胶相和凝胶 相的平衡自由能和氢键网络的一些统计特征进行了 讨论. 结果表明, 凝胶中的氢键数和凝胶自由能在临 界点附近与凝胶分数具有相同的标度行为, 并满足 同样的标度律. 此外，我们分析了氢键网络中的分子 内氢键和分子间氢键，给出了凝胶相中分子内氢键 数的计算方案, 然后以 $\mathrm{A}_{2} \mathrm{D}_{2}$ 体系为例, 直观地表明 了临界点后体系环键分数的变化趋势.

最后，我们简单强调一下氢键网络中环结构或 者说分子内氢键的重要性, 这也是本文研究氢键网 络的主要动机之一. 对于实际的氢键簇合物或网络 而言, 环键的出现将大大增益簇合物和网络的寿命, 这对于很多与氢键有关的物理化学过程都有重要影 响. 例如, 在双链 DNA 中互补碱基对之间的氢键导 致了 DNA 的稳定性, 这一特征在生物学上具有特殊 意义，同时也体现了氢键网络中分子内氢键的合作 性. 另外, 在氢键液体中氢键网络寿命的增加可为 液-固相变提供相对稳定的 “核”, 以使固相不断增长. 特别是对于典型的氢键液体一一水而言, 若体系处于 几何相变温度以下, 由于凝胶相中的氢键网络具有
类冰结构, 氢键网络还可使液态水表现出很多不同 寻常的特征. 这主要是因为水中的氢键网络包含很 多以六元环为主的环结构. 因为一个这样的环结构 会导致体系的体积增加一个小量, 加之环键和环结 构之间是一一对应的关系, 因此平均来讲, 环键的出 现意味着体积会稍有增加, 这可以解释常态水的密 度反常. 一些详细的研究已经将相应的关系定量化 ${ }^{1)}$, 并可解释有关实验数据. 当然, 在很多实际的问题中, 例如，在基于氢键而形成的组装膜和超分子液晶中， 氢键网络的作用表现出多样性、复杂性和协同性等特 征，因此也需要更加具体的分析.

\section{参考文献}

1 Bellisent-Funel M C, Dore J C. Hydrogen Bond Networks. Dordrecht: Kulumer Academic Publishers, 1994. 61—68

2 Matsumoto M, Saito S, Ohmine I. Molecular dynamics simulation of the ice nucleation and growth process leading to water freezing. Nature, 2002, 416: 409-413[DOI]

3 Geiger A, Stanley H E. Low-density "patches" in the hydrogen-bond network in liquid water. Phys Rev Lett, 1982, 49: 1749-1752[DOI]

4 Kalinichev A G, Bass J D. Hydrogen bonding in supercritical water. J Phys Chem, 1997, A101: 9720—9727

5 Stanley H E, Blumberg R L, Geiger A. Gelation models of hydrogen bond networks in liquid water. Phys Rev, 1983, B26: 16261629

6 Blumberg R L, Stanley H E, Geiger A, Mausbach P. Connectivity of hydrogen bonds in liquid water. J Chem Phys, 1984, 80: $5230-5241$

7 Tanaka F. Theory of thermoreversible gelation. Macromolecules, 1989, 22: 1988-1994[DOI]

8 Shoji M, Tanaka F. Theoretical study of hydrogen bonded supramolecular liquid crystals. Macromolecules, 2002, 35: 7460 -7472[DOI]

9 Semenov A N, Rubinstein M. Thermoreversible gelation in solutions of associative polymers. 1. Statics. Macromolecules, 1998, 31: 1373-1385[DOI]

10 Rubinstein M, Semenov A N. Thermoreversible gelation in solutions of associative polymers. 2. Linear dynamics. Macromolecules, 1998, 31: 1386-1397[DOI]

11 Wang L, Cui S, Wang Z, Zhang X, Jiang M, Chi L, Fuchs H. Multilayer assemblies of copolymer PSOH and PVP on the basis of hydrogen bonding. Langmuir, 2000, 16: 10490-10494[DOI]

12 Hao E, Lian T. Layer-by-layer assembly of CdSe nanoparticles based on hydrogen bonding. Langmuir, 2000, 21: 7879—7881[DOI]

1) Wang H J. A sol-gel model for the hydrogen bonding network in water. Phys Rev E, to be published 
13 Kato T, Mizoshita N, Kanie K. Hydrogen-bonded liquid crystalline materials. Macromol Rapid Commun, 2001, 22: $797-$ 814[DOI]

14 王海军. $\mathrm{A}_{a} \mathrm{D}_{d}$ 型氢键液体的统计理论(I): 几何相变. 中国科学, B 辑, 2006, 36(3): 211-217

15 唐敖庆, 李泽生, 孙家钟, 汤心䝠. 一般的 $\mathrm{A}_{a} \mathrm{~B}_{b}$ 缩聚反应的固 化理论. 中国科学, B 辑, 1989, 21(5): 449-466

16 李泽生, 肖兴才, 孙家钟, 唐敖庆. 高分子固化分布函数的内 禀不变性. 中国科学, B 辑, 1993, 23(9): 897-904

17 Xiao X C, Li Z S, Sun C C, Tang A C. Invariant property of the distribution of $\mathrm{A}_{a_{1}}, \cdots, \mathrm{A}_{a_{s}}-\mathrm{B}_{b_{1}}, \cdots, \mathrm{B}_{b_{1}}$ type ideal polymerization. Macromolecules, 1995, 28: 2738-2744[DOI]

18 Xiao X C, Li Z S, Sun C C, Tang A C. Intrinsic symmetry of the
$\mathrm{A}_{a} \mathrm{~B}_{b}$ type distribution. Macrolmol Theo Simul, 1994, 3: 601$606[\mathrm{DOI}]$

19 Li Z S, Ba X W, Sun C C, Tang X Y, Tang A C. $A_{a}-B_{b}$ type Stockmayer distribution and scaling study. Macromolecules, 1991, 24: 3696-3699[DOI]

20 Wang H J, Hong X Z, Zhao M, Li Z S. Statistical parameters for hydrogen bonding networks. Chin Phys Lett, 2001, 18: 764$766[\mathrm{DOI}]$

21 唐敖庆. 高分子反应统计理论. 北京: 科学出版社, 1981. 69145

22 肖兴才, 黄旭日, 王海军, 李泽生, 唐敖庆. 非线性缩聚反应的 树状胶和环状胶的反应程度. 中国科学, B 辑, 1998, 28(2): 97107 